\title{
QPOs in CVs: An executive summary
}

\author{
Brian Warner and Patrick A. Woudt \\ Dept. of Astronomy, University of Cape Town, Private Bag X3, Rondebosch 7701, South Africa
}

\begin{abstract}
An overview is given of the properties of the various kinds of quasi-periodic luminosity modulations observed in cataclysmic variables (CVs). The two principal types, known in the CV literature as dwarf nova oscillations and quasi-periodic oscillations, have similarities to the high and low frequency quasi-periodic oscillations in X-Ray binaries. There is a further well observed class known as longer period dwarf nova oscillations. In CVs the observed interrelations between these oscillations suggests a model of magnetically controlled accretion onto a rapidly rotating equatorial belt of accreted gas. Non-radial oscillations of the central white dwarf are observed in some systems.
\end{abstract}

Keywords: Stars, interacting binary; cataclysmic variables; dwarf novae; oscillations PACS: $97.10 . \mathrm{Sj}$; 97.30.Qt; $97.80 . \mathrm{Gm}$

\section{INTRODUCTION}

In many respects, the QPOs observed in cataclysmic variables (CVs) parallel what are seen in X-Ray binaries (XRB). The two fields have evolved almost independently, with slightly different terminologies, but striking similarities in properties exist. In order to inform the XRB community in a concise way about the CV QPOs we provide a manual of phenomenology, together with our current interpretation in terms of magnetic accretion. A review of CVs in general is given in [1]; a review of CV QPOs is given in [2]; here we update the latter with results partly taken from our papers $[3,4,[5,6,6,8]$, and including unpublished observations.

The story began in 1954 when Merle Walker [9] discovered highly stable 71 s coherent brightness oscillations in DQ Her, the remnant of Nova Herculis 1934. Despite a search, with the photometric techniques then available he could not find any further rapid pulsations in CVs. The advent of pulse counting photometry and application of Fourier transforms (FTs) enabled low amplitude pulsations to be dragged from the flickering noise inherent in the CV mass transfer process [10], showing that they are in fact quite common and almost exclusively connected with high mass transfer $(\dot{M})$ discs i.e. among dwarf novae during outburst and among nova-like variables (which are CVs permanently in outburst).

At about the same time improved photometric observations of DQ Her showed that observed phase and amplitude variations of the $71 \mathrm{~s}$ pulsations during eclipse [11] can be modeled as anisotropic radiation from the white dwarf primary sweeping around and 'illuminating' the concave surface of the accretion disc (e.g. [12]). A few years later $\mathrm{CVs}$ with magnetic primaries were discovered, from their optical polarization and X-Ray emission, and termed "polars" if the field on the primary is strong enough $\left(\sim 10^{7}-10^{8}\right.$ G) to enforce corotation with the secondary star and eliminate the formation of an accretion disc, or "intermediate polars" if the field is weaker, which allows formation of a disc, truncated at its inner edge by the magnetosphere of the primary. It was then realized 


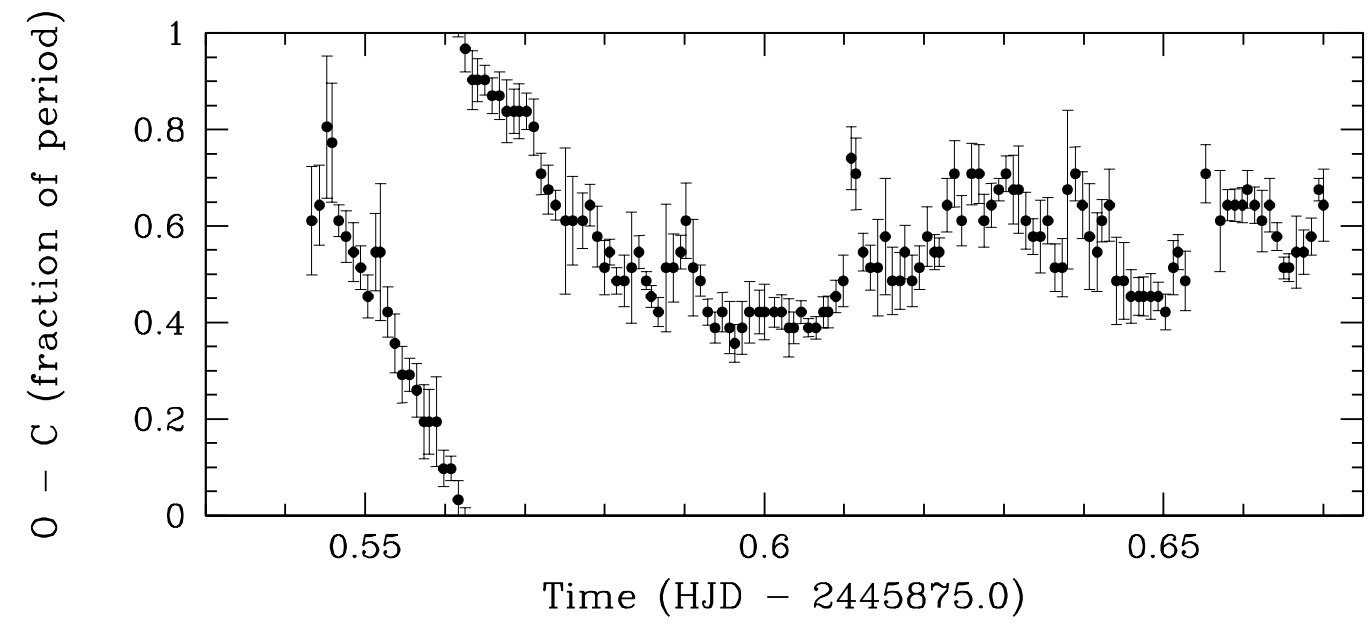

FIGURE 1. Phase variation of a 26.64-s DNO in TY PsA during outburst (run S3362: [14]).

that DQ Her was the first of the intermediate polars, and the anisotropic radiation arises in an accretion zone on the primary; the optical radiation is reprocessed hard radiation from the accretion zone. Note that intermediate polars are the white dwarf equivalents of LMXBs; there are no polar equivalents among LMXBs - their magnetic moments are too small. About $5 \%$ of isolated white dwarfs show fields over the range $10^{3}-10^{8} \mathrm{G}$ [13].

The QPOs observed in CVs are of at least four kinds, with a range of stabilities and periods. We shall describe them in order of discovery.

\section{DWARF NOVA OSCILLATIONS}

The first QPOs to be found were those in dwarf novae in outburst [10] and as a consequence they became known as dwarf nova oscillations (DNOs). They have close similarities to the high frequency QPOs in XRBs. Their properties can be summarized as follows:

- They have periods usually in the range $5-40 \mathrm{~s}$, with a characteristic range for each CV. For example, the DNO fundamental period in SS Cyg is 6-11 s.

- There is a period-luminosity relationship, in the sense that minimum period corresponds to maximum accretion luminosity, i.e. maximum $\dot{M}$ onto the primary. Thus DNO periods decrease on the rising branch of a dwarf nova outburst and increase on the descending branch.

- There are sudden small changes in period during the systematic variations $-\sim 0.03$ $\mathrm{s}$ - but no concomitant detectable luminosity changes. Unlike intermediate polars, which have $Q=\left|\frac{d P}{d t}\right|^{-1} \sim 10^{12}$, DNOs typically have $10^{3}<Q<10^{7}$. Fig. 11 shows an example of an $\mathrm{O}-\mathrm{C}$ plot of phase as a function of time.

- Although typically of amplitude $<1 \%$ in the optical, and detectable only in FTs, occasionally they are directly observable in the light curve. Fig. 2 gives an example 

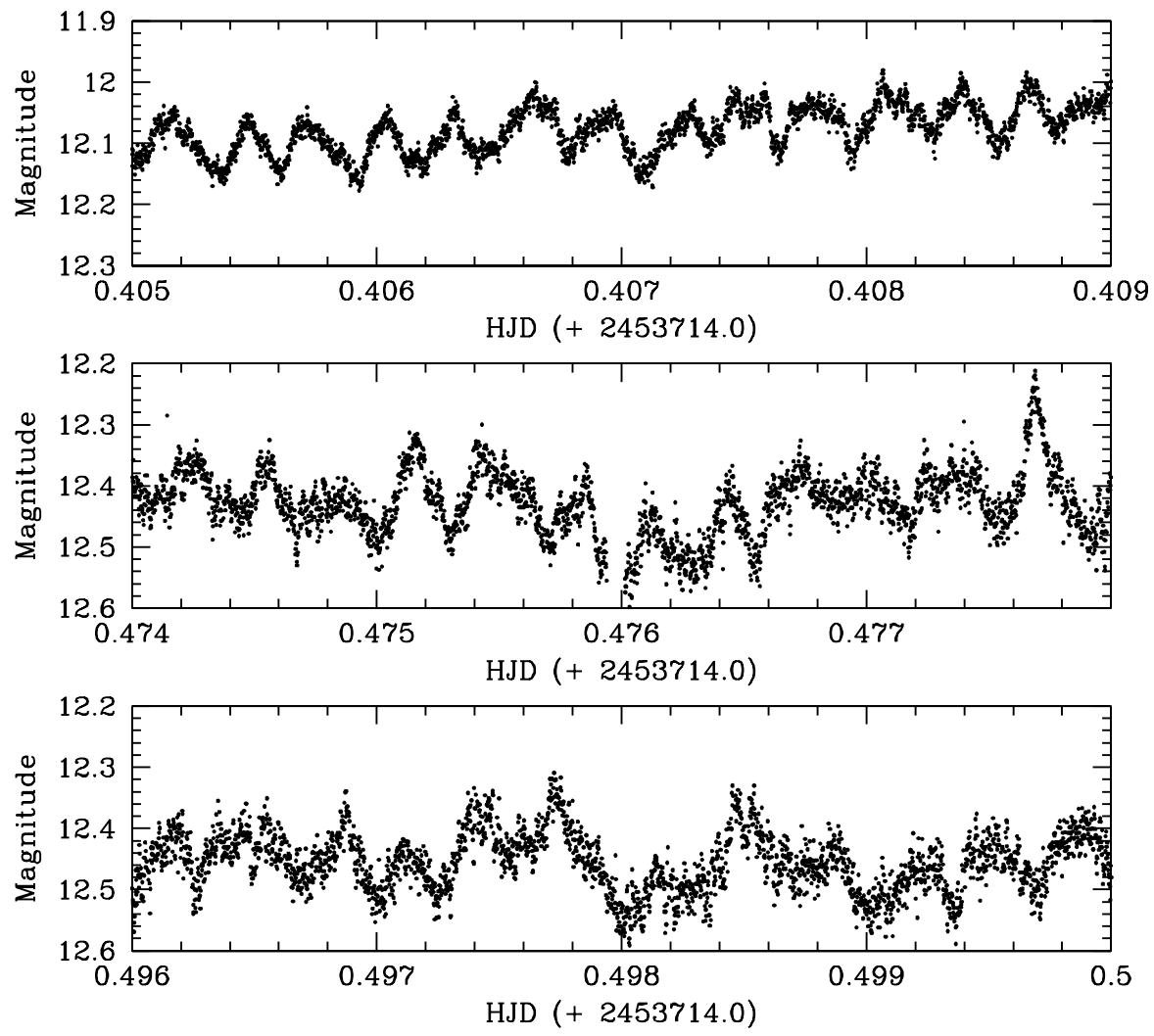

FIGURE 2. DNOs in VW Hyi during outburst observed with SALT at $80 \mathrm{~ms}$ time resolution.

of DNOs in VW Hyi during outburst, as observed with the 11-m SALT reflector. The good signal to noise enables the phase of individual pulses to be measured (as in Fig. 1). In FTs of lengthy runs of DNOs no first harmonic is detected, but Fig. 2 shows that individual pulses may be far from sinusoidal.

- DNOs have been observed in soft X-rays and the EUV in a number of CVs. The amplitudes are much larger than in the (reprocessed) optical, occasionally reaching $100 \%$, showing that a large fraction of the accreting gas may pass through the modulation machine.

- In SS Cyg at the maximum of some outbursts there is a sudden frequency doubling, with the period dropping from $6 \mathrm{~s}$ to $3 \mathrm{~s}$. In a few other dwarf novae the DNO signal disappears altogether around maximum and reappears later.

- The dwarf nova VW Hyi has been especially well studied (it is circumpolar in the southern hemisphere and outburst roughly once a month). It is unique in showing large amplitude DNOs during the final decline and into the first day of quiescence. DNOs during maximum are very rare; the minimum period is $14.1 \mathrm{~s}$, observed both in optical and soft X-Rays. There is a sequence of behaviour, repeated every outburst, during which the fundamental DNO period increases from $\sim 22 \mathrm{~s}$ to $\sim 100$ s during $\sim 30$ hours, but frequency doubling and tripling occur. During the latter parts of this evolution the fundamental rarely is present: there are often first and 


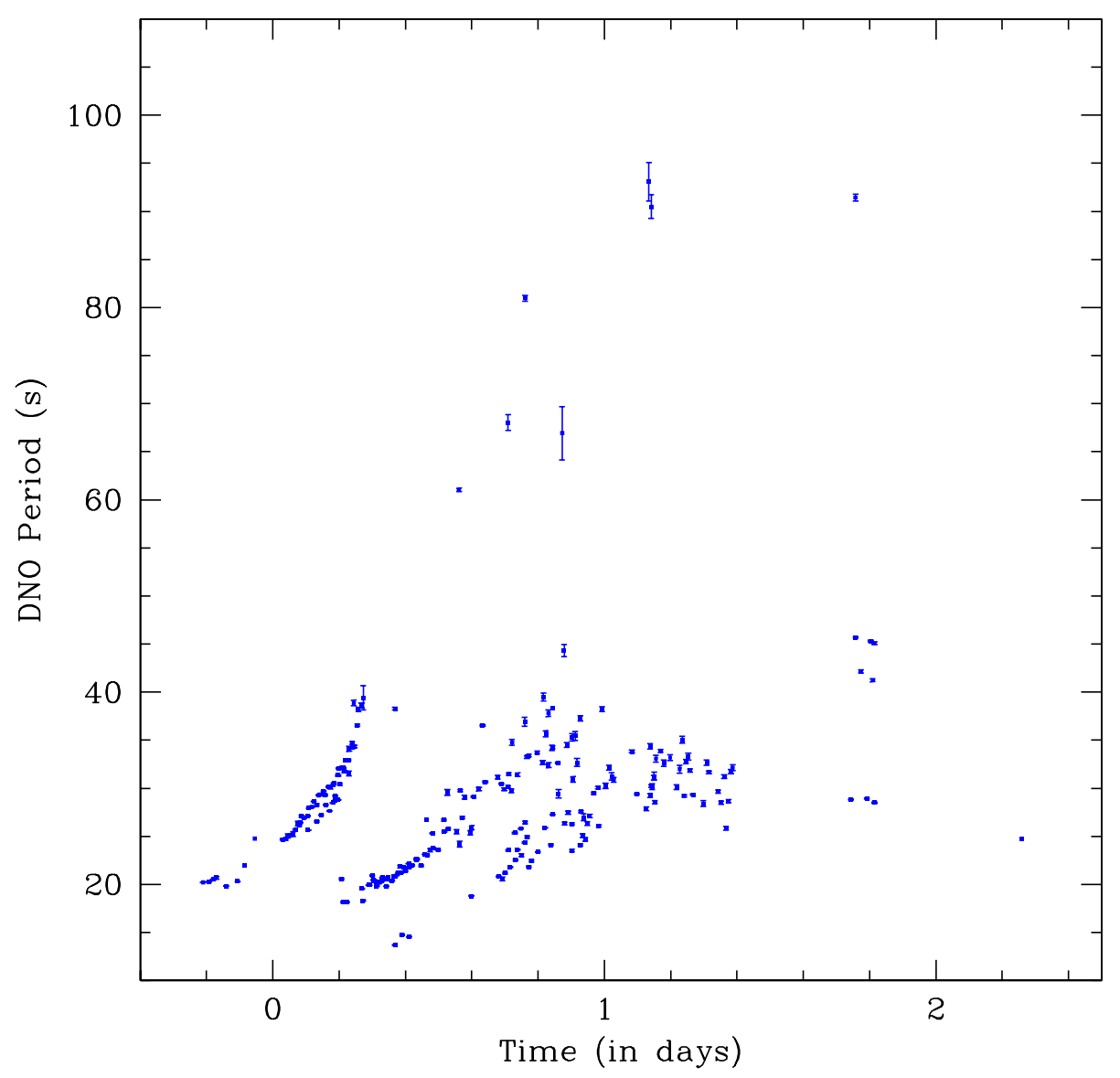

FIGURE 3. The evolution of the DNO periods at the end of normal and super outbursts in the dwarf nova VW Hyi. The zero point of the abscissa is determined from a well-defined averaged template light curve [3].

second harmonics appearing simultaneously, giving a 3:2 period ratio. Fig. 3 shows the evolution of DNOs in VW Hyi.

- During the rapid deceleration phase in VW Hyi its EUV flux is observed to drop almost to zero.

- Occasional "double DNOs" are observed in the FTs. The DNO acquires a companion, at a slightly larger period, which often has a detectable first harmonic. Its relationship to the standard DNO is demonstrated in the next section.

- The existence of a rotating beam of high energy radiation, as in DQ Her, comes from HST spectra of the dwarf nova V2051 Oph [15].

\section{QUASI-PERIODIC OSCILLATIONS}

In 1977 it was realized that there are large amplitude oscillations visible in some CV light curves, with periods much longer than those of the DNOs, which had been overlooked 


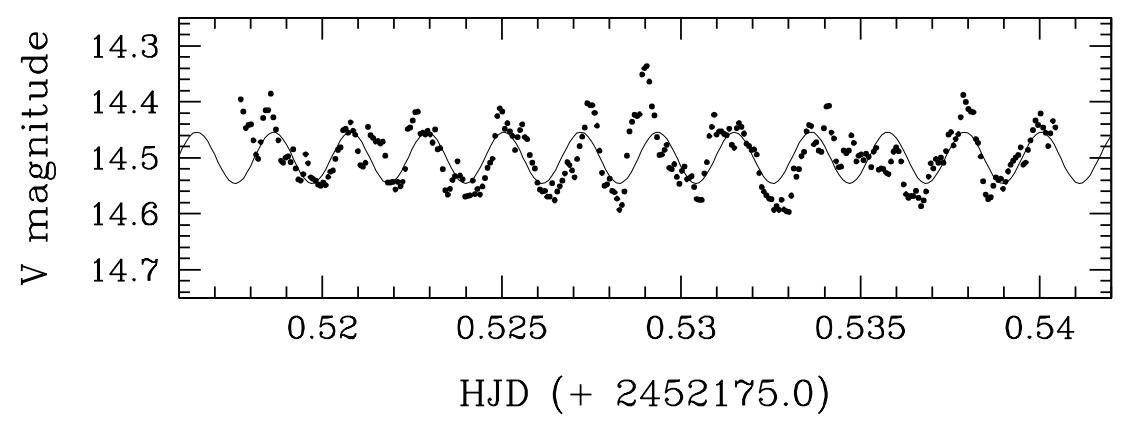

FIGURE 4. The light curve of WX Hyi showing the 185-s QPO clearly Superimposed is the result from the non-linear sinusoidal least-squares fit (reproduced from [6]).

in FTs because they have $Q \sim 5-20$ and consequently their power is spread over a wide band of frequencies [16]. Because of their overt quasi-periodicity they were designated QPOs; they have some similarities to the low frequency QPOs in XRBs.

- QPOs also commonly appear in high $\dot{M} \mathrm{CVs}$, but can exist independently of the presence of DNOs. Occasionally QPOs are seen in dwarf novae during quiescence. Fig. 4 shows an example of a large amplitude QPO that maintains phase coherence for $\sim 10$ cycles.

- Harmonics are often seen, including transitions to become entirely first harmonic.

- In only one example so far (VW Hyi) has a systematic change of period been observed during a dwarf nova outburst. The ratio $P_{\mathrm{QPO}} / P_{\mathrm{DNO}}$ maintains a value $\sim 16$ as the DNO period increases rapidly during final outburst decline.

- This ratio $P_{\mathrm{QPO}} / P_{\mathrm{DNO}} \sim 16$ is seen in many other high $\dot{M}$ systems. We think of this relationship as defining a DNO-related QPO. Fig. 51 shows an FT with DNOs and QPOs simultaneously present.

- A significant clue to the nature of the DNO-related QPOs is that in several systems the beat period of the double DNOs is equal to the observed $P_{\mathrm{QPO}}$.

- There is a second kind of QPO that have very long periods (typically $1000-2000$ s) that are less well studied.

\section{LONGER PERIOD DWARF NOVA OSCILLATIONS}

It was eventually realized that yet another QPO modulation had been reported in the literature, and observed by us, but had been previously overlooked [6]. As they lie in period between the DNOs and the DNO-related QPOs we have called them longer period DNOs (lpDNOs).

- They are observed only in high $\dot{M} \mathrm{CVs}$, both in dwarf novae during outburst and in nova-like variables.

- Occasionally they are directly observable in the light curve. 


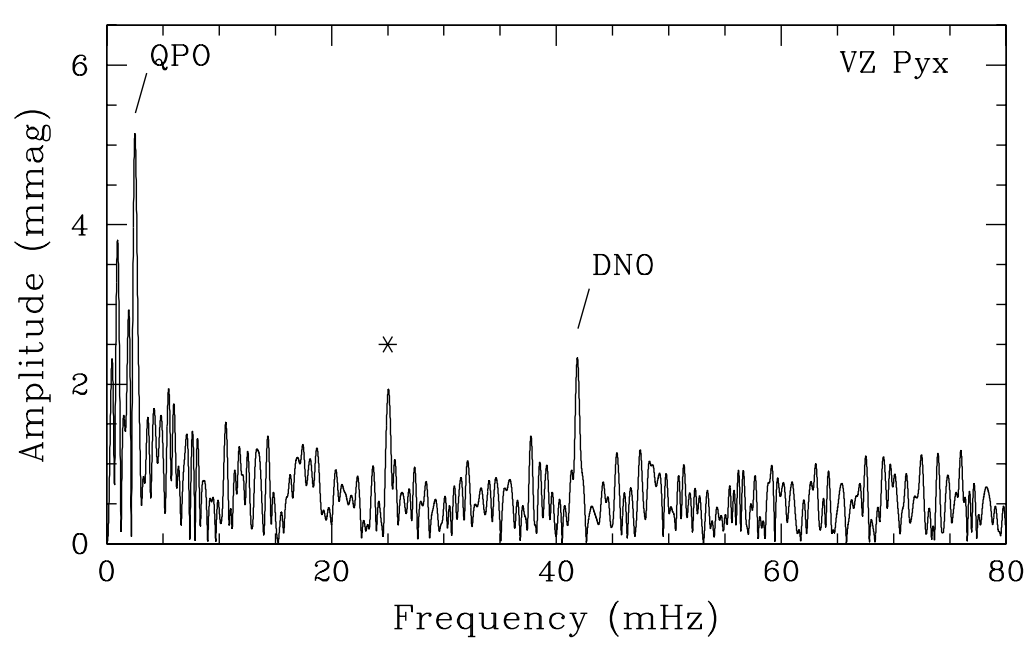

FIGURE 5. The Fourier transform of run S6569 of VZ Pyx during outburst. The QPO and DNO periods are marked. The asterisk marks a spurious signal: the telescope's periodic drive error at $40 \mathrm{~s}$ (reproduced from [6]).

- Unlike DNOs their periods are only weakly dependent of luminosity during outburst.

- It is commonly found that they have periods $\sim 4$ times those of the DNOs. So a rough relationship is $P_{\mathrm{QPO}} \sim 4 P_{\mathrm{lpDNO}} \sim 16 P_{\mathrm{DNO}}$.

- When simultaneously present, DNOs and lpDNOs show independent phase and amplitude variations.

\section{QPOs AND XRB QPOs}

By considering DNOs to be high frequency QPOs and the DNO-related QPOs to be low frequency QPOs we can plot them on the same diagram as XRB observations [6, 17]; an updated version of this graph is shown in Fig. 6. The white dwarf relationship is seen to be an extension of that for the more compact primaries - in all cases the ratio of high to low frequency QPOs is $\sim 15$.

\section{THE MODEL PROPOSED FOR CV QPOs}

So how do we explain the rich and interconnected phenomenology of CV DNOs? The most fundamental issue is the lack of stability of the periods. The inverse problem - the timing stability of white dwarfs like that in DQ Her, that have degenerate cores with almost zero viscosity - was discussed by Katz [18], who concluded that a magnetic field $\geq 10^{5} \mathrm{G}$ is required to couple the exterior to the central regions of white dwarf, resulting in rotation as a solid body, but a dwarf with $B \leq 10^{5} \mathrm{G}$ will not couple the accretion torque to the interior, allowing a rapidly rotating equatorial belt of accreted material to 


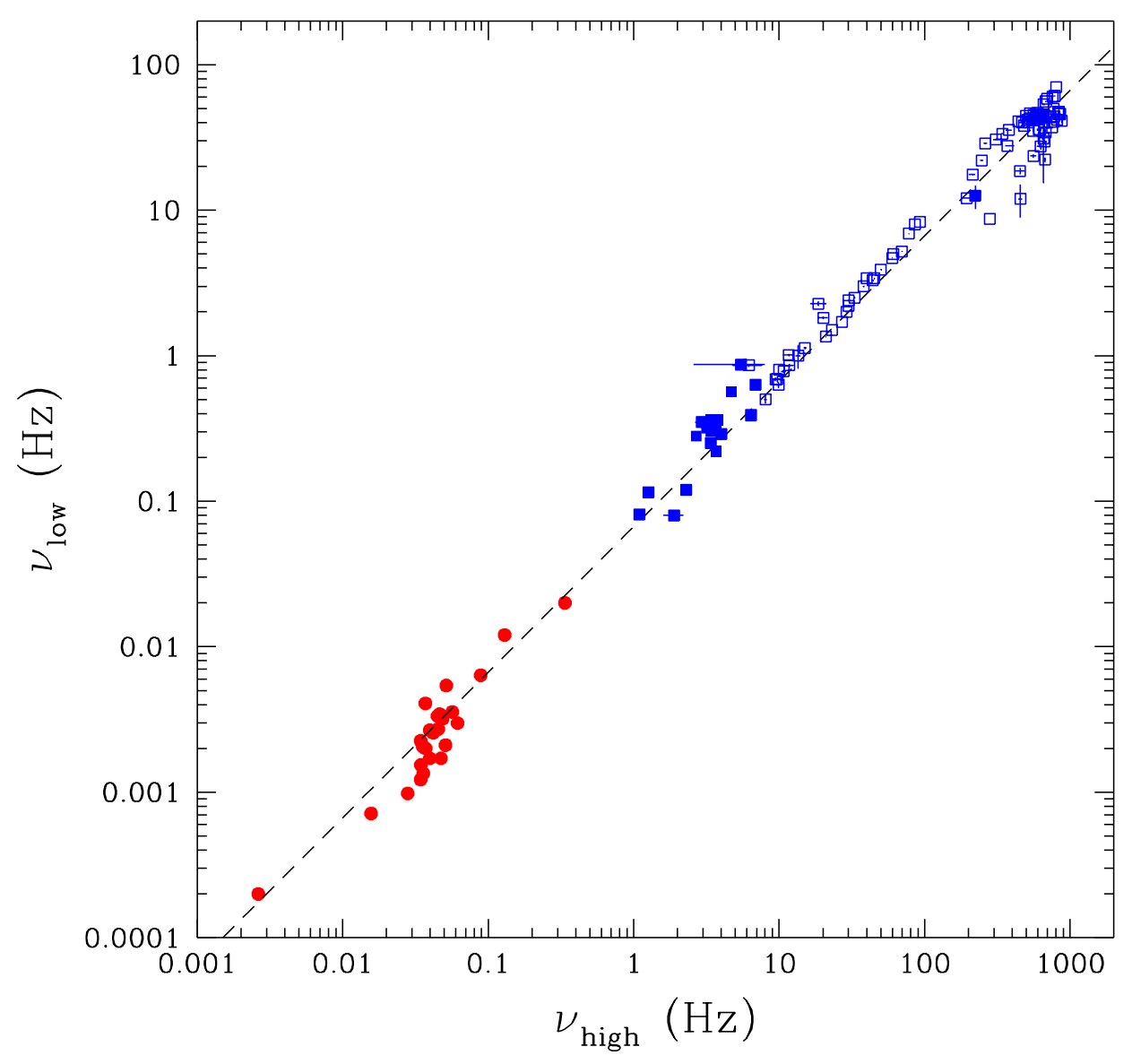

FIGURE 6. An updated version of the two-QPO diagram for X-ray binaries (filled squares: black hole binaries; open squares: neutron star binaries) and $26 \mathrm{CVs}$ (filled circles). Each CV is only shown once in this diagram. The X-ray data are from [17] and were kindly provided by T. Belloni. The dashed line marks $P_{\mathrm{QPO}} / P_{\mathrm{DNO}}=15$.

accumulate. It was this concept that suggested to Paczynski [19] that the properties of DNOs are related to the spin up and spin down of an equatorial belt. This has been elaborated, in terms of a low inertia magnetic accretor (LIMA) model (essentially a slipping intermediate polar), by Warner [20] and [4]. Evidence for hot rapidly spinning equatorial belts on dwarf novae during and after outbursts has been deduced from HST spectra [21, 22, 23, 24, 25]. The mass deposited during a typical dwarf nova outburst can be estimated from the total accretion luminosity to be $\sim 10^{22} \mathrm{~g}$, which has sufficiently low inertia to be tugged around by the field coupling it to the inner edge of the disc as the latter moves in and out in response to varying $\dot{M}$ during outburst.

The very rapid deceleration of the equatorial belt during the final stages of a VW Hyi outburst is simply the result of the inner disc radius moving out quickly as $\dot{M}$ decreases, leaving a belt rotating so rapidly that the co-rotation radius (of the belt) lies beyond the inner edge of the disc and so gas is propellered outwards into the outer disc, extracting angular momentum from the belt. The EUV flux, which is a proxy for $\dot{M}$ onto the white 
dwarf, falls as a result. Some evidence for propellering in other dwarf nova has been found [8].

We envisage that a field $\sim 10^{5} \mathrm{G}$ will act like a weak intermediate polar, as appears to be the case for WZ Sge, which has pulsations at $27.87 \mathrm{~s}$ (or harmonics) present throughout quiescence and outburst, whereas fields $\sim 10^{3-4} \mathrm{G}$ may be enhanced by differential shear in the equatorial belt during outburst, and fields $\lesssim 10^{3} \mathrm{G}$ will always be too weak to channel accretion magnetically and there will be no DNOs observed (as is indeed the case for a few well-observed dwarf novae and nova-likes).

The double DNOs and their relation to the concomitant QPOs suggest an analogy to intermediate polars. There, as well as the spin frequency $\omega$ of the white dwarf and the orbital frequency $\Omega$, a side band at $\omega-\Omega$ is often seen, the result of reprocessing of the white dwarf beamed radiation off the secondary (or any other target revolving with the orbital period). For the QPOs this required a reprocessing 'wall' rotating progradely with period $P_{\mathrm{QPO}}$, producing QPOs in the light curve by alternately partly obscuring and/or reprocessing radiation from the central part of the disc, and intersecting the rotating beam. Just such a travelling wave is shown by Lubow \& Pringle [26] to be the most likely mode to be excited at the inner edge of an accretion disc. There are a number of permutations of double DNOs and QPOs possible - we do not always see the QPO in a light curve, even if a double DNO shows that one is present, and a QPO wave observed by us is not necessarily intersected by the DNO beam to produce double DNOs.

Finally we come to the lpDNOs - what is their origin? In many respects they behave like standard DNOs, occasionally showing doubling at the QPO frequency, but being insensitive to accretion luminosity. A possible clue comes from $v \sin i$ measurements made of the primaries from HST spectra - in general the rotation periods of the primaries are twice the lpDNO period, consistent with two-pole accretion onto the body of the primary. Possibly, therefore, there is accretion from the disc via field lines that connect to the primary; alternatively, the source of the lpDNO modulation could be in the disc just outside the corotation radius [8]. The outer parts of the primary are not strongly coupled to the interior, so angular momentum of material spreading from the equatorial accretion zone will result in differential rotation as a function of latitude, perhaps resulting in the variation of $1 \mathrm{pDNO}$ period. An important clue is seen in the overall evolution of DNO and lpDNO periods in VW Hyi (Fig. 7), where the fundamental DNO period evolves towards the lpDNO period and ceases to increase when the two have converged.

\section{NON-RADIAL OSCILLATIONS OF THE PRIMARY}

In addition to the QPOs, which are clearly not situated in the body of the primary, multiple oscillations are seen in the primaries of some low $\dot{M}$ CVs. Among isolated white dwarfs four instability strips are found, within which non-radial oscillations are excited. The first and coolest of these was first recognized by [10]. The surface temperatures of $\mathrm{CV}$ primaries are largely determined by $\dot{M}$, and it happens that to be in the coolest $(\sim 11000 \mathrm{~K})$ instability strip requires an $\dot{M}$ that generates an accretion luminosity sufficiently low for the white dwarf itself not to be outshone. The first of the CVs with pulsating primaries was found in 1997 [27], since when more than a dozen more have been found (listed in [28]). Typical pulsation periods lie in the range $80-1300 \mathrm{~s}$. We 


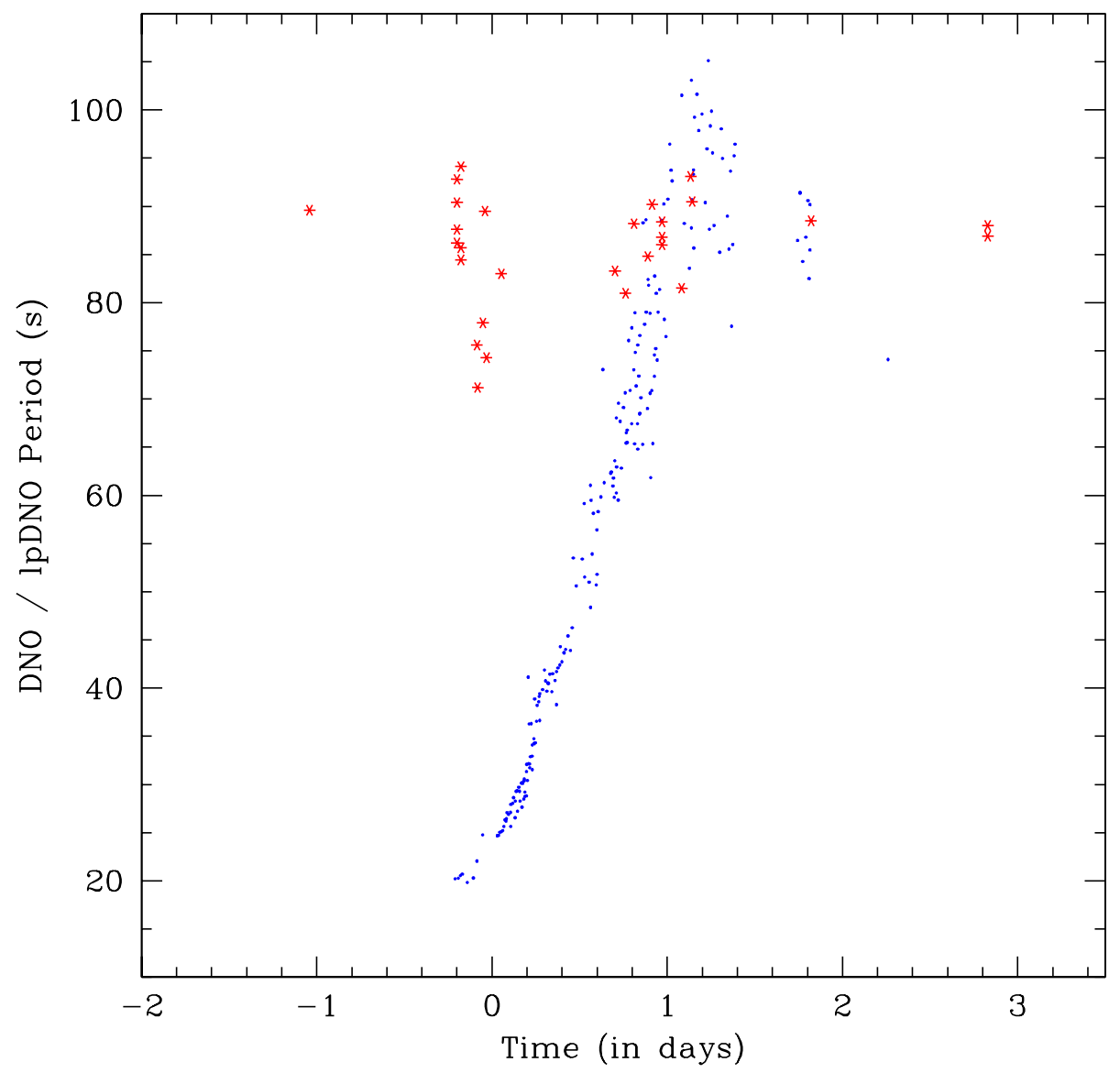

FIGURE 7. Time-evolution of the observed or implied fundamental DNO periods (small dots) and lpDNO periods (asterisks).

have recently detected what appear to be multiple pulsations in two nova remnants, one was a nova in 1986 and the other in 2007 . If confirmed, these will probably be found to lie in one of the hotter instability strips.

\section{ACKNOWLEDGMENTS}

We are grateful to the SALT team for providing the observations on which Fig. 2 is based; a full description of these will be published elsewhere. BW's research is supported by the University of Cape Town. He is grateful to the conference organisers for partial financial support that made his attendance at the conference possible. PAW's research is supported by the National Research Foundation and by the University of Cape Town. 


\section{REFERENCES}

1. B. Warner, Cataclysmic Variable Stars. Cambridge University Press (1995a).

2. B. Warner, Publ. Ast. Soc. Pac. 116, 115-132 (2004).

3. P. A. Woudt, and B. Warner, Mon. Not. R. Ast. Soc. 333, 411-422 (2002).

4. B. Warner, and P. A. Woudt, Mon. Not. R. Ast. Soc. 335, 84-98 (2002).

5. B. Warner, and P. A. Woudt, Mon. Not. R. Ast. Soc. 367, 1562-1576 (2006).

6. B. Warner, P. A. Woudt, and M. L. Pretorius, Mon. Not. R. Ast. Soc. 344, 1193-1209 (2003).

7. M. L. Pretorius, B. Warner, and P. A. Woudt, Mon. Not. R. Ast. Soc. 368, 361-370 (2006).

8. B. Warner, and M. L. Pretorius, Mon. Not. R. Ast. Soc. 383, 1469-1476 (2008).

9. M. F. Walker, Astroph. J. 123, 68-89 (1956).

10. B. Warner, and E. L. Robinson, Nature Phys. Sci. 239, 2-7 (1972).

11. B. Warner, W. L. Peters, W. B. Hubbard, and R. E. Nather, Mon. Not. R. Ast. Soc. 159, 321-335 (1972)

12. J. Petterson, Astrophys. J. 241, 247-256 (1980).

13. D. T. Wickramasinghe, and L. Ferrario, Publ. Ast. Soc. Pac. 112, 873-924 (2000).

14. B. Warner, D. O’Donoghue, \& W. Wargau, Mon. Not. R. Ast. Soc. 238, 73-96 (1989).

15. D. Steeghs, K. O’Brien, K. Horne, R. Gomer, and J. B. Oke, Mon. Not. R. Ast. Soc. 323, 484-496 (2001).

16. J. Patterson, E. L. Robinson, and R. E. Nather, Astrophys. J. 214, 144-151 (1977).

17. T. Belloni, D. Psaltis, and M. van der Klis, Astroph. J. 572, 392-406 (2002)

18. J. L. Katz, Astroph. J. 200, 298-305 (1975).

19. B. Paczyński, 1978, in Nonstationary Evolution of Close Binaries edited by A. Zytkov, Polish Sci. Publ., Warsaw, 89-98 (1978).

20. B. Warner, ASP Conf. Ser. 85, 343-354 (1995b).

21. E. M. Sion, F. H. Cheng, M. Huang, I. Hubeny, and P. Szkody, Astroph. J. Lett. 471, 41-44 (1996).

22. B. T. Gänsicke, and K. Beuermann, Astron. and Astroph. 309, L47-L50 (1996).

23. F. H. Cheng, E. M. Sion, K. Horne, I. Hubeny, M. Huang, and S. D. Vrtilek, Astron. J. 114, 1165-1169 (1997).

24. P. Szkody, D. W. Hoard, E. M. Sion, S. B. Howell, F. H. Cheng, and W. M. Sparks, Astroph. J. 497, 928-934 (1998).

25. E. M. Sion, and J. Urban, Astroph. J. 572, 456-460 (2002).

26. S. H. Lubow, and J. E. Pringle, Astroph. J., 409, 360-371 (1993).

27. B. Warner, and L. van Zyl, IAU Symp. 185, 321-322 (1998).

28. T. Marsh, http://deneb.astro.warwick.ac.uk/phsaap/pulsators.html (2006). 